\title{
Influence of slope and external temperature on smoke stratification in case of fire in bi-directional road tunnel
}

\author{
Peter Weisenpacher ${ }^{1, *}$, Jan Glasa ${ }^{1}$, and Lukas Valasek $^{1}$ \\ ${ }^{1}$ Institute of Informatics, Slovak Academy of Sciences, Dubravska cesta 9, 84507 Bratislava, Slovakia
}

\begin{abstract}
Jet fan ventilation strategy in case of fire in bi-directional road tunnels is focused on maintaining smoke stratification. There are several factors influencing stratification under specific conditions. In this paper smoke movement during a $5 \mathrm{MW}$ fire in a $600 \mathrm{~m}$ long road tunnel is studied by computer simulation and the influence of slope and external temperature on smoke stratification is analysed. Calculations were performed on a high performance computer cluster using parallel version of Fire Dynamics Simulator. Smoke stratification upstream of the fire is maintained in every simulation scenario with the exception of declivous tunnel, in which buoyancy intensifies backlayering. The behaviour of the smoke movement downstream of the fire is more complex. In the case of horizontal tunnel the stratification is not maintained in the vicinity of the fire and region with untenable conditions expands downstream. In the tunnel with slope of $-2^{\circ}$ this expansion is accelerated, while in the tunnel with slope of $2^{\circ}$ untenable conditions spread in opposite direction. The influence of exterior temperature higher than temperature inside the tunnel is relatively weak in horizontal tunnels; however, it becomes very important in sloping tunnels, especially downstream of the fire.
\end{abstract}

\section{Introduction}

In case of fire in bi-directional road tunnels people are trapped both upstream and downstream of the fire. Therefore, tenability conditions for human life should be maintained long enough to ensure safe evacuation in both parts of the tunnel tube. An air flow with critical velocity pushing smoke away from the tunnel cannot be used as the conditions downstream of the fire would become untenable for people. Instead, jet fans are used to achieve the prescribed target velocity of the flow maintaining stratification of smoke layer. Although backlayering cannot be prevented under such circumstances, the smoke layer spreads above human heads and tenable conditions for human life are preserved.

An important question connected with this strategy is the determination of a proper value of target velocity. Various national regulations recommend the values of velocity ranging from 1.0 to $1.5 \mathrm{~m} . \mathrm{s}^{-1}$ (for example [1, 2]). After the prescribed target velocity is achieved, the flow interacts with buoyancy forces created by the fire as well as with specific tunnel tube geometry. It may influence air flows in some regions of the tunnel as well as spread of smoke. Therefore, a thorough analysis is needed to evaluate specific fire scenarios. The main mechanism causing the descent of smoke layer is entrainment between the hot and cold layer [3]. However, there is a lack of knowledge for the general applicability of entrainment equations $[4,5]$. Therefore, the results of computer simulation of specific fire and ventilation scenarios can become a useful guide for tunnel management. It can be also used for training and education of tunnel specialists $[6,7]$. This problem is of special importance in Slovakia, where several road tunnels with longitudinal ventilation are currently under construction, including some bi-directional tunnels [8, 9].

In [10] we have investigated smoke movement in a 600 $\mathrm{m}$ long road tunnel by computer simulation for fire scenarios with various heat release rates. The influence of several prescribed values of the target air flow velocity on smoke stratification and visibility has been examined for chosen placement of the fire source. The simulation results indicate that it is difficult to maintain smoke stratification for fires with heat release rate (HRR) exceeding the value of $3 \mathrm{MW}$ corresponding to passenger car fire. There has been no value of the target velocity maintaining smoke stratification in both upstream and downstream parts of the tunnel found, although the $1 \mathrm{~m} . \mathrm{s}^{-1}$ velocity leads to the highest visibility achieved in evaluated sectors of the tunnel. Velocity above $1 \mathrm{~m} . \mathrm{s}^{-1}$ produces smoke eddies in the downstream part of the tunnel, while lower values of velocity result in significant backlayering upstream of the fire.

In this study, the smoke stratification in a $600 \mathrm{~m}$ long road tunnel is evaluated for selected fire scenarios with the $5 \mathrm{MW} \mathrm{HRR}$ and the air flow velocities of 1.0 and 1.5 $\mathrm{m} . \mathrm{s}^{-1}$ simulated by the Fire Dynamics Simulator (FDS), version 6.5.2 [11,12]. An influence of external temperature and the tunnel slope on maintaining smoke

* Corresponding author: upsyweis@savba.sk 
stratification is studied. Smoke movement as well as tenability criteria for the considered scenarios are evaluated in particular parts of the tunnel tube. As our research has demonstrated the applicability of parallel versions of the FDS for tunnel fire simulations [13], calculations are performed on the high-performance computer (HPC) cluster.

\section{Fire Dynamics Simulator}

The FDS is a CFD-based simulation system for modelling fire and fire-driven fluid flows. I was developed by the National Institute of Standards and Technology (USA) with cooperation with VTT Technical Research Centre of Finland (Finland) [11,12]. The FDS numerically solves a form of conservation equations for low-speed, thermally-driven flows with an emphasis on the smoke and heat transport from fire. It includes models of fire related physical and chemical processes such as turbulence, thermal radiation, pyrolysis, combustion of the pyrolysis products, conductive heat transfer, etc. The FDS enables various kinds of parallelization of calculation.

Input data required for the FDS simulation include geometry of the investigated compartment (the tunnel size and shape, location, size and shape of all objects in the tunnel), representation of jet fans, material properties (ignition temperature, thermal conductivity, specific heat, density, emissivity, heat of evaporation, etc.) and the fire HRR. The most important output quantities of the gaseous component are temperature, velocity, pressure and density of the gas, the concentration of smoke and visibility.

\section{Fire Scenarios}

We use the FDS model of the $600 \mathrm{~m}$ long bi-directional road tunnel. The tunnel has circular cross section and is equipped with one pair of jet fans (see Fig. 1).

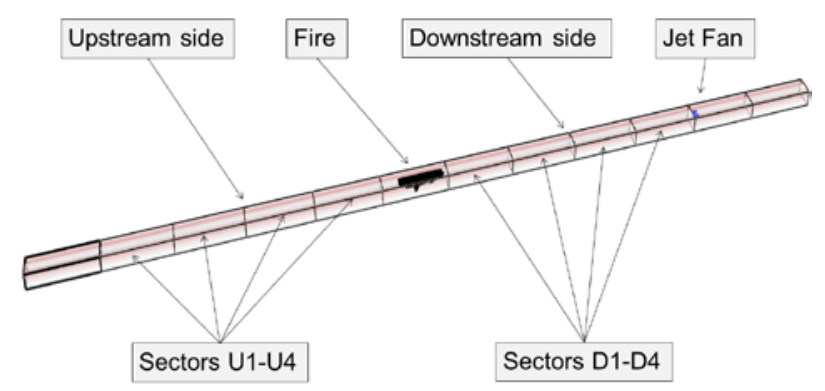

Figure 1. Scheme of the tunnel.

Dimensions of the cross section of the tunnel are 10.8 $\mathrm{m}$ width, $6.8 \mathrm{~m}$ height. Cross section area of jet fans is $0.5 \mathrm{~m}^{2}$, their length is $3.7 \mathrm{~m}$. These parameters are typical for tunnels which are currently under construction in Slovakia. The distance of jet fans pair from the left portal is $503.2 \mathrm{~m}$. The tunnel includes two regions in which stratification is evaluated (on both sides of the fire). Each of them is approximately $200 \mathrm{~m}$ long.
The length of tunnel region necessary for dissipation of jet fan airflow may exceed $100 \mathrm{~m}$. Therefore, the length of the tunnel is sufficient to describe all considered phenomena related with smoke stratification realistically.

The fans are represented as an HVAC, using rectangular vents of dimensions $0.6 \mathrm{~m} \mathrm{x} 0.8 \mathrm{~m}$ with prescribed normal velocity. The volume flow of the jet fans at the beginning of the simulation is $15 \mathrm{~m}^{3} . \mathrm{s}^{-1}$. After the required target velocity of the air flow in the tunnel is reached, the performance of jet fans decreases with the aim to stabilize the flow velocity at a prescribed value. Two different values of the target velocity are tested: 1.0 and $1.5 \mathrm{~m} . \mathrm{s}^{-1}$. The initial value of air velocity in the tunnel is $0 \mathrm{~m} \cdot \mathrm{s}^{-1}$.

The fire source is modelled by a rectangular block placed $276 \mathrm{~m}$ from the left tunnel portal. Its top surface $\left(4 \mathrm{~m}^{2}\right)$ burns with the heat release rate per unit area (HRRPUA) of $1250 \mathrm{~kW} \cdot \mathrm{m}^{-2}$ producing the $5 \mathrm{MW}$ fire. The fire HRR increases linearly, reaching its maximal value at the $40^{\text {th }} \mathrm{s}$ of the fire. The fire soot yield is 0.2 .

The smoke distribution is evaluated in eight sectors of the $50 \mathrm{~m}$ length (see Fig. 1). Four of them (U1 - U4) are located in the upstream part of the tunnel, other four in the downstream part (D1 - D4). Somewhere in this area emergency exits would be situated in a real tunnel; therefore, maintaining the tenability conditions for human life $[14,15]$ in these sectors would be the aim of tunnel management. Tenability conditions require the visibility at human head level above $10 \mathrm{~m}$. In each sector the visibility at head level $(1.6 \mathrm{~m})$ averaged within its whole area is evaluated during the $420 \mathrm{~s}$ fire. This quantity provides only simplified aggregated measure of smoke distribution; however, it describes main trends of the smoke movement sufficiently.

Several fire scenarios are investigated for two values of target velocity. In the scenarios V1.0 and V1.5 for the target velocity of 1.0 and $1.5 \mathrm{~m} . \mathrm{s}^{-1}$, respectively, the empty horizontal tunnel and the $10^{\circ} \mathrm{C}$ ambient temperature within the tunnel are assumed. In the scenario V1.x-C 20 passenger cars are distributed on both sides of the fire. In the scenario $V 1 . x-E$, the influence of modification of external temperature to $15^{\circ} \mathrm{C}$ is studied. The scenario V1.0-Es with the same parameters includes also the simulation of $2000 \mathrm{~s}$ before the start of the fire in order to achieve the realistic stabilized velocity profile induced by different internal and external temperatures at the beginning of the fire. In the V1.x-Sl-2 and V1.x-Sl2 scenarios, the slope of the tunnel is $-2^{\circ}$ and $2^{\circ}$, respectively. In the latter one the opposite direction of jet fan flow is necessary to vanquish buoyancy forces created by the fire. Therefore, the different location of the jet fans (48 $\mathrm{m}$ from the left portal) is used to prevent a direct interaction of their jets with smoke. The V1.x-Sl2E and V1.x-Sl2Es scenarios evaluate the influence of the $15^{\circ} \mathrm{C}$ external temperature for the tunnel with $2^{\circ}$ slope. In the latter scenario, a stabilization of the air flow in the tunnel before the fire start is also simulated.

The computational domain size is $600 \mathrm{~m} \mathrm{x} 12 \mathrm{~m} \mathrm{x}$ $7.2 \mathrm{~m}$. For the $20 \mathrm{~cm}$ mesh resolution, the domain consists of $3000 \times 60 \times 36$ cells. The total number of 


\begin{tabular}{|c|c|c|c|c|c|c|c|c|c|c|}
\hline Scenario & $\begin{array}{c}\text { U1 } \\
{[\mathrm{m}]}\end{array}$ & $\begin{array}{l}\mathrm{U} 2 \\
{[\mathrm{~m}]}\end{array}$ & $\begin{array}{l}\mathrm{U} 3 \\
{[\mathrm{~m}]}\end{array}$ & $\begin{array}{r}\text { U4 } \\
{[\mathrm{m}]}\end{array}$ & $\begin{array}{l}\text { D1 } \\
{[\mathrm{m}]}\end{array}$ & $\begin{array}{l}\text { D2 } \\
{[\mathrm{m}]}\end{array}$ & $\begin{array}{l}\text { D3 } \\
{[\mathrm{m}]}\end{array}$ & $\begin{array}{l}\text { D4 } \\
{[\mathrm{m}]}\end{array}$ & $\begin{array}{c}\text { Jet } \\
{[\mathrm{m} / \mathrm{s}]}\end{array}$ & $\begin{array}{c}\mathrm{Vel} \\
{[\mathrm{m} / \mathrm{s}]}\end{array}$ \\
\hline$V 1.0$ & 30,0 & 30,0 & 30,0 & 29,3 & 4,7 & 7,9 & 15,0 & 21,0 & 11,0 & 1,0 \\
\hline$V 1.0-C$ & 30,0 & 30,0 & 30,0 & 30,0 & 4,8 & 6,0 & 14,1 & 22,8 & 13,1 & 1,0 \\
\hline$V 1.0-E$ & 30,0 & 30,0 & 28,0 & 26,7 & 3,8 & 6,4 & 16,3 & 23,9 & 10,9 & 1,0 \\
\hline V1.0-ES & 30,0 & 30,0 & 28,0 & 26,9 & 4,1 & 7,0 & 15,1 & 20,7 & 11,1 & 1,0 \\
\hline V1.0-Sl2 & 30,0 & 30,0 & 30,0 & 30,0 & 23,2 & 28,6 & 5,5 & 1,3 & $-8,0$ & 1,0 \\
\hline V1.0-Sl2E & 30,0 & 30,0 & 30,0 & 29,7 & 24,4 & 18,8 & 2,0 & 1,1 & $-11,1$ & 1,0 \\
\hline V1.0-Sl2Es & 30,0 & 30,0 & 30,0 & 29,8 & 26,0 & 14,5 & 2,4 & 1,1 & $-12,6$ & 1,1 \\
\hline V1.0-Sl-2 & 29,8 & 24,5 & 7,4 & 2,2 & 1,1 & 0,9 & 0,9 & 0,9 & 21,4 & 1,0 \\
\hline$V 1.5$ & 30,0 & 30,0 & 30,0 & 30,0 & 3,2 & 3,1 & 3,6 & 3,8 & 14,1 & 1,5 \\
\hline$V 1.5-C$ & 30,0 & 30,0 & 30,0 & 30,0 & 4,7 & 4,6 & 3,8 & 3,9 & 16,7 & 1,5 \\
\hline$V 1.5-E$ & 30,0 & 30,0 & 30,0 & 29,8 & 3,0 & 2,9 & 3,3 & 3,7 & 14,1 & 1,5 \\
\hline V1.5-Es & 30,0 & 30,0 & 30,0 & 29,8 & 2,6 & 2,9 & 3,2 & 3,5 & 14,2 & 1,5 \\
\hline$V 1.5-S l 2$ & 30,0 & 30,0 & 30,0 & 30,0 & 7,5 & 9,1 & 12,0 & 20,7 & 6,2 & 1,5 \\
\hline V1.5-Sl2E & 30,0 & 30,0 & 30,0 & 30,0 & 6,3 & 8,2 & 10,7 & 19,1 & $-3,8$ & 1,5 \\
\hline V1.5-Sl2ES & 30,0 & 30,0 & 30,0 & 30,0 & 6,1 & 8,4 & 14,9 & 18,1 & $-6,9$ & 1,5 \\
\hline
\end{tabular}

Figure 2. Average visibility in the evaluated sectors U1 - U4 and D1 - D4 in the upstream and downstream part of the tunnel during the last $20 \mathrm{~s}$ of the fire; jet fan air flow velocity and velocity averaged within the whole tunnel cross section for all considered scenarios.

cells is $6,480,000$. In order to increase the simulation performance the parallel MPI version 6.5.2 of the FDS is used. The computational domain was decomposed into 12 meshes, each of them assigned to one MPI process (one CPU core). Eight of these meshes correspond to sectors U1 - U4 and D1 - D4.

All simulations were carried out on the SIVVP HPC cluster at the Institute of Informatics, Slovak Academy of Sciences, Bratislava (Slovakia) [16]. It is an IBM dx360 M3 cluster consisting of 54 computational nodes (23 Intel E5645@ 2.4 GHz CPU, 48 GB RAM); the total number of cores is 648 . The nodes are connected by the Infiniband interconnection network with the bandwidth of $40 \mathrm{Gbit} / \mathrm{s}$ per link and direction. The total CPU time depends on specifications of each fire scenario. The CPU time varies from 79 hours for the scenario $V 1.0-S l 2$ to 278 hours $(V 1.5-C)$.

\section{Simulation Results}

The results of the simulations are consistent with [10]. Fig. 2 shows average visibility in the evaluated sectors in the upstream and downstream region of the tunnel during last $20 \mathrm{~s}$ of the fire for all considered scenarios. The colours of the cells indicate tenable or untenable conditions during considered period. It can be seen that the effect of passenger cars placed in the tunnel as well as the effect of different exterior temperature on the visibility in horizontal tunnel is relatively weak. However, the effect of the slope as well as contribution of the temperature differences between internal and external temperature to this effect are considerable.

\subsection{Smoke stratification upstream of the fire}

A significant backlayering occurs in all tested scenarios, although the length of backlayering strongly depends on simulation parameters. However, the smoke stratification upstream of the fire is well maintained in every simulation scenario with the exception of the scenario with the $-2^{\circ}$ tunnel slope. Smoke layer upstream of the fire for selected scenarios can be seen in Fig. 3.

As can be expected, higher target velocities result in shorter lengths of backlayering. Moreover, in the scenario $V 1.0$ a slight drop of visibility occurs, while in $V 1.5$ a faster air flow maintains a perfect visibility. The cars located in the tunnel have a similar effect. As the average velocity within the tunnel cross section at the beginning of the tunnel is kept constant, the flow velocity above cars roofs must be increased to fulfil the mass conservation equation, which decelerates the movement of the smoke layer in opposite direction. Faster air flow also prevents a slight decrease of visibility which can be observed in scenario V1.0. The effect of temperature differences between internal and external temperatures is more pronounced than Fig. 2 indicates. Warmer air flowing into the tunnel decreases the temperature gradient between hot and cold layer dissolving smoke layer and increasing entrainment. However, a small drop of visibility in these scenarios does not constitute a threat for people, as tenability conditions are fulfilled.

In the simulations $V 1 . x$-Sl2 buoyancy forces suppress backlayering even more while in the scenario $V 1 . x-S l-2$ backlayering is increased. In the latter scenario buoyancy increases the velocity of smoke layer. As the average velocity within the tunnel cross section is constant, the 
velocity of flow above the road in opposite direction must be increased as well, thus intensifying entrainment, dissolving smoke layer and disrupting smoke stratification. This is the reason of a lower visibility in this scenario. In two of the four evaluated sectors (U3 and U4) the visibility drop causes conditions untenable for human lives.
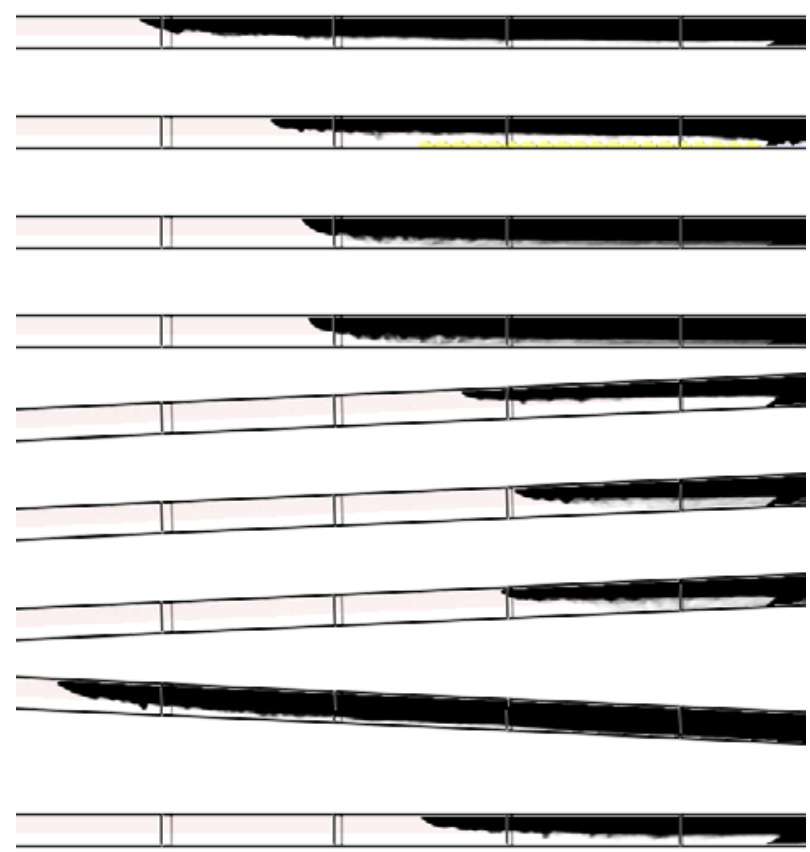

Figure 3. Smoke layer upstream of the fire after $400 \mathrm{~s}$ for the scenarios V1.0, V1.0-C, V1.0-E, V1.0-Es, V1.0-Sl2, V1.0-Sl2E, V1.0-Sl2Es, V1.0-Sl-2 and V1.5.

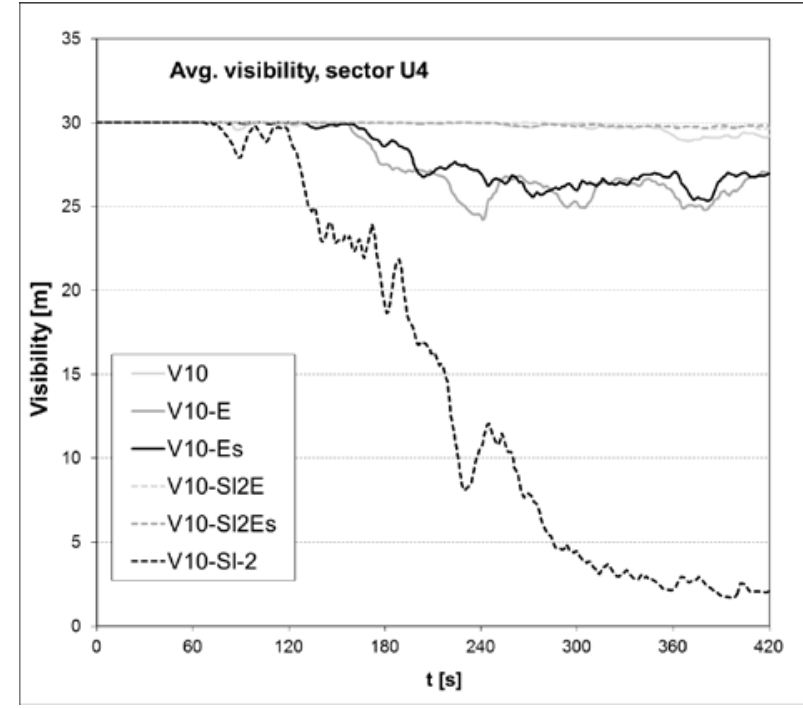

Figure 4. Time behaviour of averaged visibility in the sector U4 for selected scenarios

Differences between scenarios with or without the 2000 second stabilisation period are very small. The influence of natural flow of warmer air inside the tunnel at the beginning of the fire in the scenarios with stabilization is negligible in comparison with the influence of warm air flowing into the tunnel as a consequence of jet fan activity.

In all tested scenarios in which the visibility drop is observed it occurs in sectors U3 and U4. Time behaviour of averaged visibility in critical sector U4 for scenarios where perfect visibility upstream of the fire is not maintained can be seen in Fig. 4. Initial drop of visibility occurs after 2-3 minutes to the fire. Such behaviour is consistent with the results of [10].

In the same way, the higher target velocity is more efficient in maintaining the smoke stratification upstream of the fire. Only very small visibility decrease occurs in the case of some scenarios with the $1.5 \mathrm{~m} . \mathrm{s}^{-1}$ target velocity in sector $\mathrm{U} 4$.

\subsection{Smoke stratification downstream of the fire}

None of the tested values of the target velocity in any scenario is able to maintain smoke stratification in all evaluated sectors downstream of the fire (see Fig. 2). However, time behaviour as well as the final values of the averaged visibility in the sectors D1 - D4 are very different for particular scenarios (see Figures 5-8).

The slope of the tunnel has the greatest impact on smoke movement. The drop of visibility and descent of the smoke layer in horizontal tunnel typically starts in the vicinity of the fire (sector D1) due to interaction of the fire with the air flow and spreads downstream to the other sectors [10]. The target velocities higher than 1.0 m.s ${ }^{-1}$ intensifies this effect (see Figures 2 and 5). This pattern can be seen also in Fig. 6. In the sectors D3 and D4 tenability conditions are fulfilled until the end of the simulation, unlike the scenario V1.5.

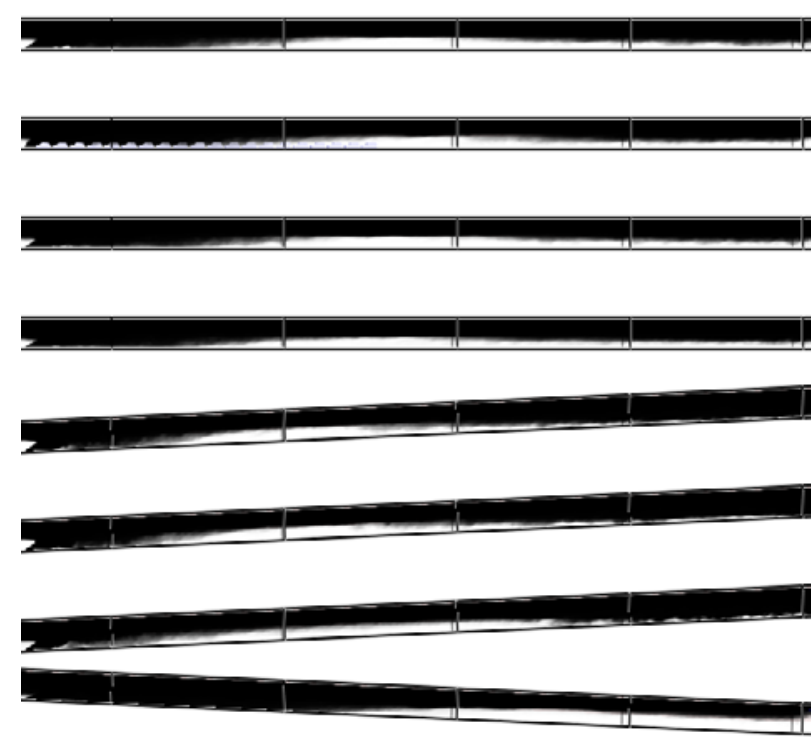

2

Figure 5. Smoke layer downstream of the fire after $150 \mathrm{~s}$ for scenarios V1.0, V1.0-C, V1.0-E, V1.0-Es, V1.0-Sl2, V1.0-Sl2E, V1.0-Sl2Es, V1.0-Sl-2 and V1.5. 


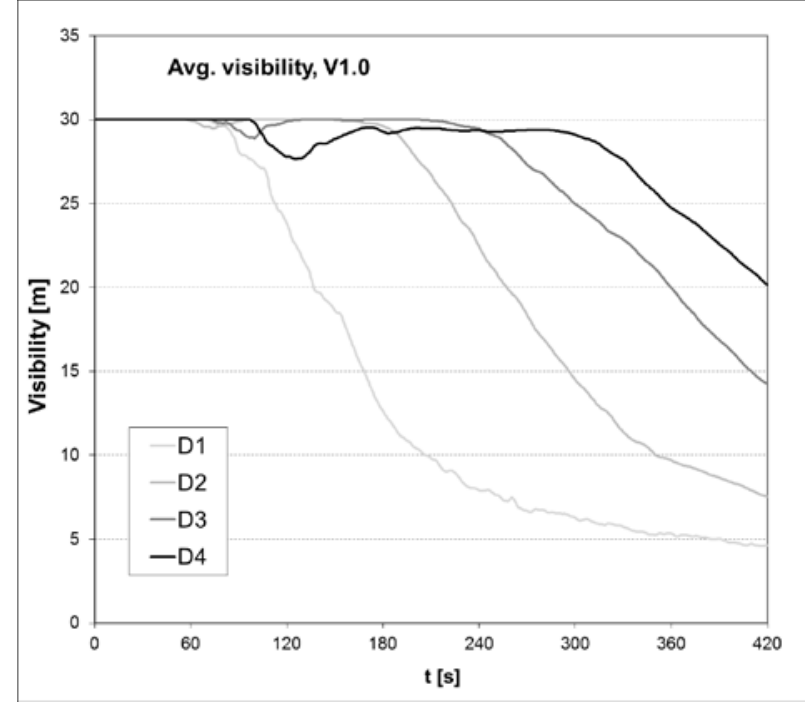

Figure 6. Time behaviour of averaged visibility in sectors D1 - D4 in scenario V1.0.

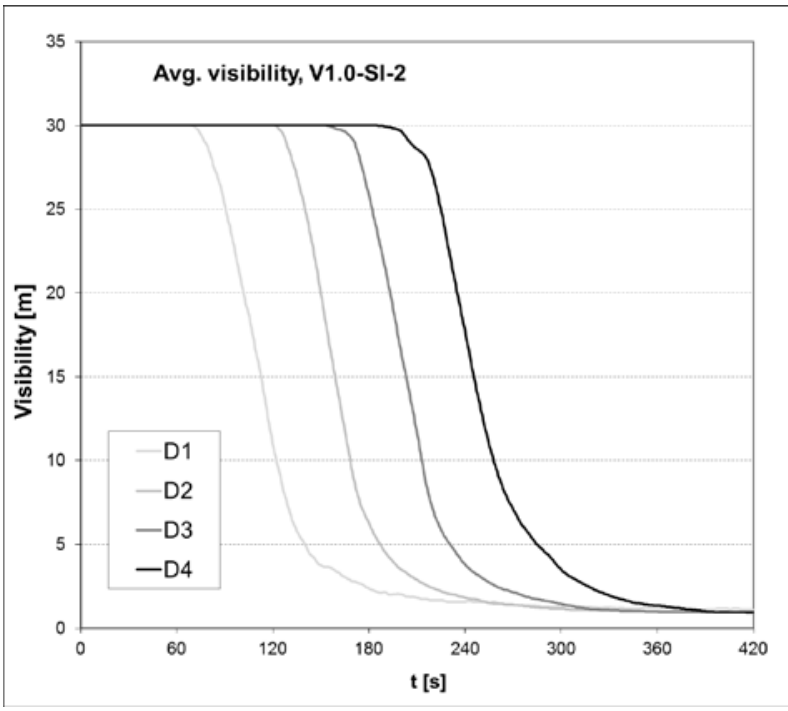

Figure 7. Averaged visibility in scenario $\mathrm{V1.0}-\mathrm{Sl}-2$.

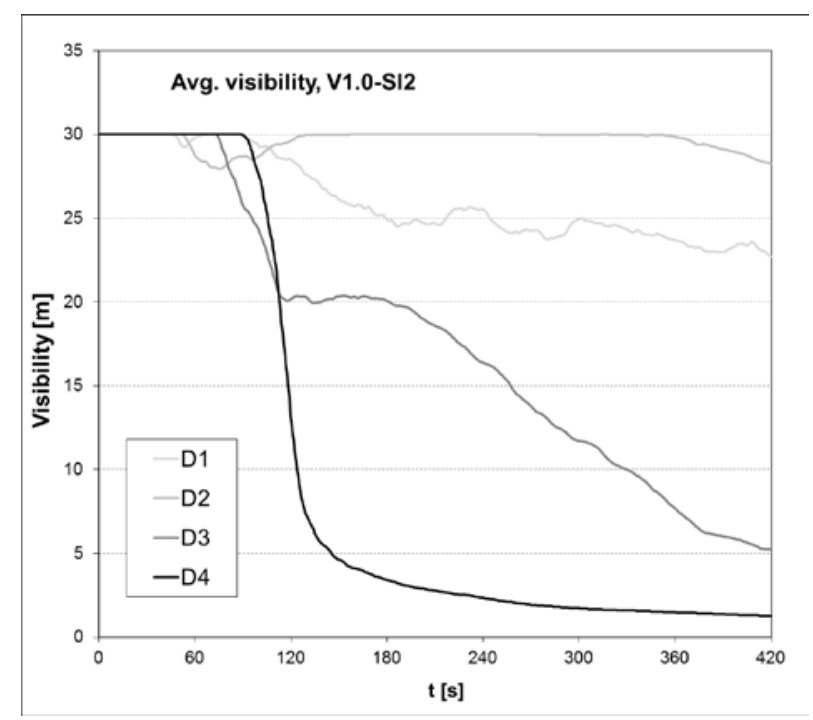

Figure 8. Averaged visibility in scenario V1.0-Sl2.
In the tunnel with the slope of $-2^{\circ}$ visibility drops faster than in the horizontal tunnel under the same circumstances. Until the $5^{\text {th }}$ minute of the fire conditions for human life become untenable in all the sectors (see Fig. 7). The reason of this behaviour is buoyancy which increases an air flow velocity in front of the fire. It has a similar effect as a significant increase of target velocity. In such conditions the smoke stratifications cannot be maintained.

In the tunnel with the slope of $2^{\circ}$ the smoke behaviour is more complex (see Fig. 8). There are two mechanisms decreasing visibility. In the sector D1 it is a direct interaction of the air flow with the fire. It is the same mechanism as in the scenarios discussed above; however, it is attenuated due to the effect of buoyancy which decreases the air velocity in front of the fire. This is the reason of tenability conditions in the sectors D1 and D2 being well-preserved until the end of the simulation. However, buoyancy increases the movement of smoke under the tunnel ceiling downstream of the fire and thus also entrainment. It results in a considerable dissolution of the smoke layer in the sector D4 and in somewhat less pronounced dissolution in the sector D3. Tenability conditions in these sectors are not fulfilled at the end of simulation. The slope of the tunnel changes the course of the smoke layer dissolution completely.

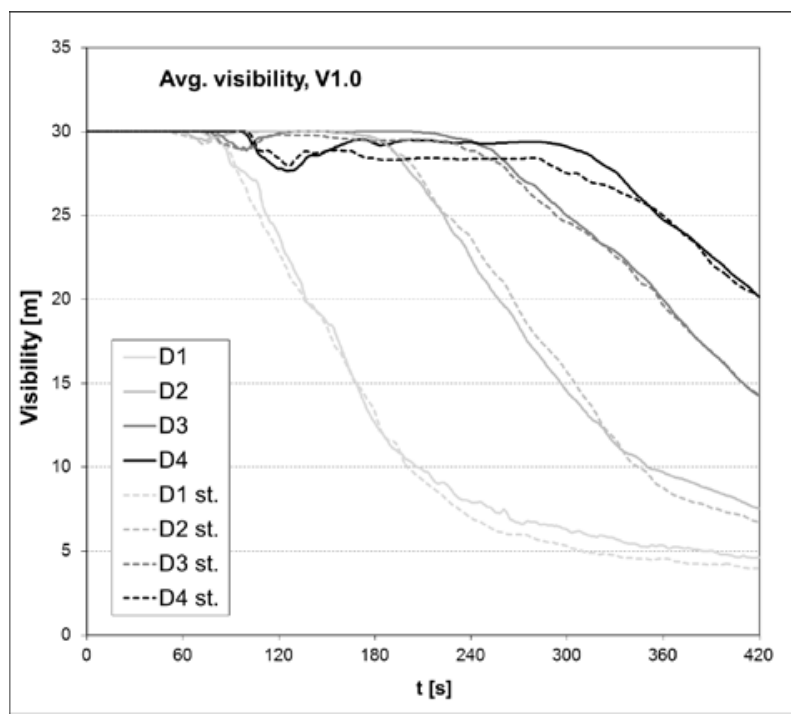

Figure 9. Averaged visibility in scenario V1.0 (with and without stabilisation).

Although the effect of exterior temperature in horizontal tunnel is relatively weak (see Fig. 9), there is a considerable impact of the temperature gradient on smoke movement in sloping tunnel (see Fig. 10). In V1.0-Sl2ES scenario a combination of the slope and temperature gradient creates a flow of warmer air under the tunnel ceiling at the beginning of the fire, which does not exist in non-stabilized scenario. Although the higher temperature under the ceiling decelerates dissolution of the smoke layer, it simultaneously increases the difference between the velocities under the ceiling and above the road surface and thus it increases the entrainment. 


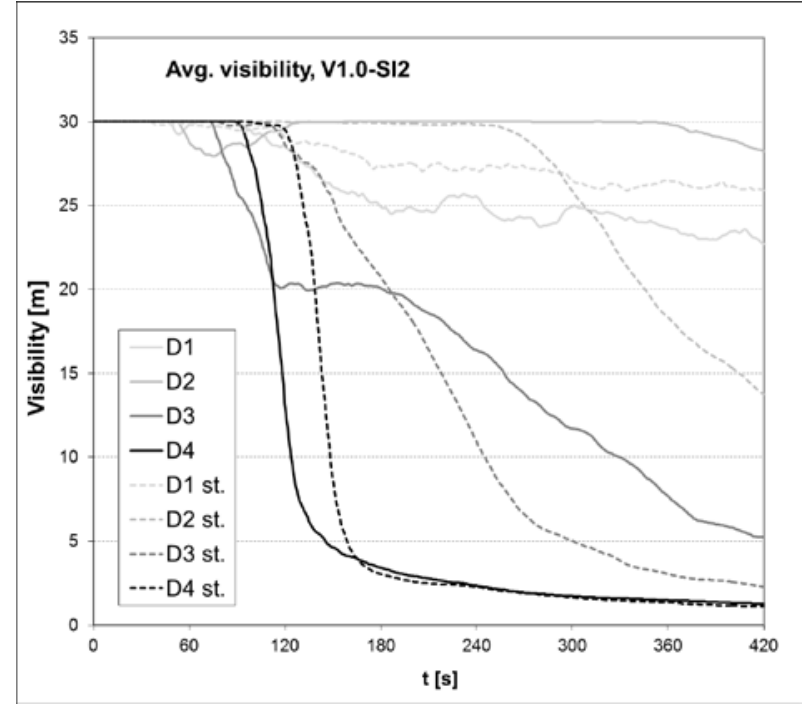

Figure 10. Averaged visibility in scenario V1.0-Sl2 (with and without stabilisation).

In the early phase of the burning, the first of above mentioned mechanisms prevails in the sectors D3 and D4 resulting in a better visibility in the stabilized scenario. After approximately 3 minutes of the fire the influence of natural warm flow becomes negligible in comparison with the heat produced by the fire and the effect of a more intensive entrainment prevails. The visibility in stabilized scenarios becomes worse. In the sector D2 the latter mechanism prevails during the whole simulation. The sector D1 is the only one in which the visibility in the stabilized scenario is better than in the non-stabilized.

\section{Conclusions}

In this paper the smoke movement during $5 \mathrm{MW}$ tunnel fire is simulated by the FDS, version 6.5.2 for selected fire scenarios. The influence of slope and external temperature on the smoke stratification is investigated for two different values of the target air flow velocity.

The smoke stratification upstream of the fire is well maintained in every simulation scenario with the exception of the scenario with the $-2^{\circ}$ tunnel slope, in which buoyancy strongly increases backlayering. The influence of temperature differences on the smoke stratification is relatively weak. The first drops of visibility typically start in the sectors in the vicinity of the fire.

The most important factor influencing stratification downstream of the fire is slope. In horizontal tunnel the visibility drop starts in the vicinity of the fire and spreads downstream. In the tunnel with the slope of $-2^{\circ}$ this spread is accelerated, while in the tunnel with the slope of $2^{\circ}$ the region with untenable conditions expands in the opposite direction. If the exterior temperature is higher than the temperature inside the tunnel, visibility is deteriorated even more significantly.

\section{Acknowledgements}

The authors would like to thank P. Schmidt, B. Spisakova (National Motorway Company) for information about technical specifications of road tunnels and M. Dobrucky, J. Astalos and V. Sipkova for technical support for SIVVP HPC cluster computing. This paper was partially supported by the Slovak Science Foundation (project No. VEGA 2/0165/17) and the Slovak Research and Development Agency (project No. APVV-15-0340).

\section{References}

[1] TP 12/2011 Road tunnels ventilation (in Slovak), (Ministry of Transport and Construction of the Slovak Republic, 2011)

[2] Guideline - Road tunnels ventilation (in Czech), (Ministry of Transport of the Czech Republic, 2013)

[3] H. Ingason, Y.Z. Li, A. Lönnermark, Tunnel fire dynamics (Springer-Verlag, New York, 2015, p. 321)

[4] R.L. Alpert, Fire induced turbulent ceiling-jet (Report no. 19722-2. Factory Mutual Research Corp., Norwood, 1971)

[5] H.Z. You, G.M. Faeth, Fire and Materials. 9, 46 (1985)

[6] P. Danisovic, J. Sramek, E. Jancarikova, Proceedings of 9th annual International Conference of Education, Research and Innovation, Seville, Spain (2016)

[7] E. Jancarikova, P. Danisovic, J. Sramek, Proceedings of 5th International Conference "Integration, Partnership and Innovation in Construction Science and Education”, Moscow, Russia (2016)

[8] http://ndsas12.cmsromboid.sk/prehladtunelov/44346s

[9] http://www.sta-itaaites.sk/index.php?page=tunely_vo_vystavbe

[10] P. Weisenpacher, J. Glasa J, L. Valasek, Proceedings of 10th Mediterranean Combustion Symposium, Naples, Italy (in press)

[11] K. McGrattan, S. Hostikka, R. McDermott, J. Floyd, C. Weinschenk, K. Overholt, Fire Dynamics Simulator, Technical Reference Guide (National Institute of Standards and Technology, Gaithersburg, Maryland, USA, and VTT Technical Research Centre of Finland, Espoo, Finland, sixth edition, 2017)

[12] K. McGrattan, S. Hostikka, R. McDermott, J. Floyd, C. Weinschenk, K. Overholt, Fire Dynamics Simulator, User's Guide (National Institute of Standards and Technology, Gaithersburg, Maryland, USA, and VTT Technical Research Centre of Finland, Espoo, Finland, sixth edition, 2017)

[13] P. Weisenpacher, J. Glasa, L. Halada, L. Valasek, V. Sipkova, Comput. Inform. 331237 (2014)

[14] Practice Note for Tenability Criteria in Building Fires (Society of Fire Safety, Engineers Australia, 2014)

[15] D.A.Purser, Assessment of Hazards to Occupants from Smoke, Toxic Gases and Heat (In: P. J. DiNenno (Ed.), The SFPE Handbook of Fire Protection 
Engineering. 4th Edition, National Fire Protection Association, Quincy, MA 02269, 2009, pp. 2-96 - 2-193)

[16] SIVVP - Slovak Infrastructure for High

Performance Computing. Available from:

http://www.sivvp.sk/, http://hpc.ui.savba.sk/ 